\title{
Médiévales
}

Langues, Textes, Histoire

50 | printemps 2006

Sociétés nordiques en politique (XII ${ }^{\mathrm{e}}-\mathrm{XV}^{\mathrm{e}}$ siècles)

\section{Marguerite, ses officiers et la lettre d'instructions à Erik de Poméranie (1405)}

Queen Margaret and her officials, and the letter she adressed to Erik of

Pomerania in 1405

\section{Raphaëlle Schott}

\section{OpenEdition}

Journals

Édition électronique

URL : https://journals.openedition.org/medievales/1367

DOI : 10.4000/medievales. 1367

ISSN : 1777-5892

Éditeur

Presses universitaires de Vincennes

Édition imprimée

Date de publication : 1 juin 2006

Pagination : 103-120

ISBN : 2-84292-186-0

ISSN : 0751-2708

\section{Référence électronique}

Raphaëlle Schott, « Marquerite, ses officiers et la lettre d'instructions à Erik de Poméranie (1405) »,

Médiévales [En ligne], 50 I printemps 2006, mis en ligne le 15 septembre 2008, consulté le 24 avril

2022. URL : http://journals.openedition.org/medievales/1367 ; DOI : https://doi.org/10.4000/

medievales. 1367

Ce document a été généré automatiquement le 24 avril 2022.

Tous droits réservés 


\section{Marguerite, ses officiers et la lettre d'instructions à Erik de Poméranie (1405)}

Queen Margaret and her officials, and the letter she adressed to Erik of

Pomerania in 1405

Raphaëlle Schott

1 Dans Le chemin de longue étude, Christine de Pizan dépeint quatre dames assises sur des trônes correspondant aux quatre points cardinaux. La Sagesse siège à l'est, la Chevalerie au sud, la Richesse à l'ouest et la Noblesse au nord :

Me tournant de l'autre côté, je vis en retrait un second trône disposé pour marquer

le Septentrion (...). Sur cette belle chaire se tenait une dame couronnée, au maintien noble et distingué (...). La dame tenait un sceptre à la main ; à ses pieds, elle avait placé un grand roi, qui se prosternait matin et soir pour lui servir d'appui ${ }^{1}$.

2 L'image est forte et n'est pas sans ressembler à la situation politique des royaumes nordiques autour de 1400, que Christine de Pizan connaissait peut-être. L'allégorie de la noblesse du Chemin de longue étude et la présence effective, en Scandinavie, d'une souveraine qui était parvenue à porter à la tête d'un triple royaume son petit-neveu, Erik de Poméranie, couronné roi de l'Union en 1397 à Kalmar, présentent des corrélations troublantes. Le gouvernement de la reine Marguerite fut-il perçu en Occident comme un modèle politique ? Cette hypothèse ne prend-elle pas tout son sens lorsqu'on analyse la lettre que cette dernière adressa au roi en 1405 et que l'historiographie scandinave a coutume d'appeler la « lettre d'instructions ${ }^{2}$ »?

3 Cette lettre, écrite en ancien danois, se compose de cinquante-quatre paragraphes qui sont autant de recommandations que Marguerite, forte de son expérience politique, fit à Erik sur le point de partir pour la Norvège. Usant du style tantôt direct, tantôt indirect, la reine prit soin de transcrire littéralement les propos que le jeune roi serait susceptible d'échanger avec ses officiers lors de son tour du royaume. Ces dialogues anticipés mettent en scène une succession de situations conflictuelles, auxquelles le 
jeune homme ne pourrait faire face qu'en relisant «souvent ces lignes et articles de manière à en saisir pleinement le sens ${ }^{3}$ ". Ce discours à double voix permet d'entrevoir la complexité des relations de pouvoir entre le souverain et l'aristocratie locale. D'une part, les réclamations des officiers témoignent de l'ambiguïté de leur comportement, dans la mesure où ces serviteurs dévoués à la Couronne étaient aussi les représentants d'un groupe social soucieux de défendre ses intérêts. D'autre part, les répliques dictées mot pour mot à Erik mettent en lumière les ambiguïtés propres à l'exercice du pouvoir : comment déléguer une partie de la souveraineté royale sans en perdre le contrôle? Quand faire confiance et quand se méfier? Quand flatter et quand réprimander? Les différents aspects de ce dialogue mettent en évidence une lutte d'influence entre deux partis qui, paradoxalement, ne sauraient exister l'un sans l'autre. D'un côté en effet, le pouvoir royal, pour affirmer sa souveraineté, devait pouvoir confier à des officiers des territoires plus ou moins vastes que ces derniers administraient en son nom. De l'autre, les seigneurs tiraient prestige et rémunération du service au roi, tout en cherchant à accroître leur fortune et leurs privilèges au détriment de ce dernier.

Deux thèmes reviennent de manière récurrente : d'une part, l'octroi et la restitution des forteresses et des terres de la Couronne confiées en administration (sysle et len) ${ }^{4}$ et de l'autre, la perception des impôts. La reine, qui séjournait pour l'essentiel au Danemark, entendait vérifier qu'elle était toujours maîtresse en son royaume héréditaire de Norvège. Comme l'écrit Bernard Guenée, «le premier souci d'un prince qui se veut puissant à la fin du Moyen Âge est de restaurer son domaine, de le réformer en envoyant des enquêteurs-réformateurs révoquer les aliénations abusives, pourchasser les officiers incompétents et malhonnêtes et raviver tous ses droits ${ }^{5}$ ». Le document étudié présente une situation relativement insolite, puisque l'officier envoyé comme « enquêteur-réformateur » n'est autre que le roi en personne.

Dans un premier temps, je présenterai dans ses grandes lignes l'appareil administratif norvégien au sein duquel coexistaient deux systèmes, l'un favorable à une forte centralisation monarchique, l'autre aux intérêts personnels des officiers en place. Je dresserai dans un second temps une typologie des malversations et abus contre lesquels Marguerite mit le jeune roi en garde. Ses soupçons, dont il n'est pas toujours possible de vérifier le bien-fondé, sont intéressants dans la mesure où ils révèlent un rapport de force entre la Couronne et l'aristocratie. Enfin, j'étudierai les solutions envisagées par Marguerite pour faire valoir ses droits et renforcer ses positions. Je m'interrogerai en conclusion sur les stratégies politiques qui se dessinent derrière ces règlements administratifs.

Le système des sysler et la politique royale des len

6 Le terme de sysle désignait à l'origine la mission (ombud) dont les hommes du roi étaient investis localement. Ce terme prit progressivement une connotation territoriale et fut couramment utilisé au $\mathrm{XIII}^{\mathrm{e}}$ siècle pour désigner les grands districts administratifs royaux ${ }^{6}$. Au siècle suivant, la Norvège ${ }^{7}$ comptait une cinquantaine de ces sysler, à la tête desquels des officiers (syslemenn) agissaient comme des représentants du roi et exerçaient en son nom une autorité militaire, judiciaire et fiscale. Ces officiers étaient chargés de prélever les impôts, taxes et amendes pour le compte de la Couronne. Ces collectes étaient ensuite versées aux fehirder, officiers en poste dans les quatre grandes villes du royaume (Trondheim, Bergen, Oslo et Tønsberg ${ }^{8}$ ). Les sysler organisés autour d'une forteresse (slottslen) étaient administrés par des syslemenn qui étaient aussi des capitaines de château, et qui étaient signalés dans les sources par les termes 
d'høvedsmenn ou encore de fogder (bailli). À partir du règne de Håkon V (1299-1319), probablement par souci de sécurité, les centres de perception furent couplés avec les grandes forteresses royales et les capitaines d'Akershus, Tønsberg, Bohus et Bergen remplirent du même coup les fonctions des fehirder ${ }^{9}$. En échange de leurs services, le roi autorisait ces hommes à prélever leur salaire sur les amendes royales. Piliers de l'appareil administratif norvégien, ils sont donc au cœur des préoccupations exprimées par la reine dans sa lettre.

7 La mise en place du système des sysler accompagna la centralisation du pouvoir monarchique en Norvège. Il s'agissait d'une forme d'administration domaniale (kronoförvaltning ${ }^{10}$ ), dont Marguerite énonce clairement le principe au paragraphe 29 :

Si Tideke ${ }^{11}$ réclame ce skipreide ${ }^{12}$ afin de pouvoir laisser sortir ses chevaux, qu'[Erik] lui réponde que ce skipreide ne lui sera confié en len ni pour ses chevaux, ni pour une autre raison, mais comme rétribution de son service, qui durera aussi longtemps que sa Grâce le dira.

Le seigneur qui recevait un sysle en len était donc investi d'un service pour le compte du roi, et non d'une terre à titre privé ${ }^{13}$. Le lensmannsreversen, c'est-à-dire le serment que le syslemann devait prêter par écrit au roi au moment de son investiture, témoigne du même principe. L'officier devait promettre de gérer au mieux les propriétés de la Couronne et de ne tirer aucun avantage personnel de son office. Il s'engageait à reverser les sommes collectées en temps et en heure, à rendre une comptabilité annuelle et à prélever l'exacte rétribution qui lui revenait en droit ${ }^{14}$.

9 Il est difficile de savoir si les termes de len et de sysle recouvraient la même signification. Bien que les sources les utilisent parfois comme synonymes, les historiens établissent en général une distinction entre le terme de len, correspondant vraisemblablement à la rétribution de l'officier, et celui de sysle, désignant en priorité l'entité administrative ${ }^{15}$. Cette ambivalence terminologique pose donc la question de la part "publique » et de la part «privée » des sysler, et plus largement du caractère féodal $\mathrm{du}$ système administratif norvégien. Erik Opsahl pense que la société norvégienne médiévale ne correspondait pas au schéma de féodalité traditionnel européen, mais répondait davantage à une forme de "féodalité bâtarde ». Les relations entre le roi et ses vassaux n'étaient pas fondées sur un hommage et sur une terre à caractère héréditaire, mais sur un contrat de service aux aspects financiers fortement marqués. Le serviteur bénéficiait du soutien et de la protection de ses intérêts par le roi ; le roi renforçait son prestige et s'assurait de l'alliance politique et militaire de ses représentants locaux ${ }^{16}$.

10 Le système des sysler, où les len étaient donc accordés en l'échange d'un service et soumis à reddition des comptes (len på regnskap), prévalut aux $\mathrm{XIII}^{\mathrm{e}}$ et $\mathrm{XIV}^{\mathrm{e}}$ siècles en Norvège. Il fut néanmoins en concurrence avec d'autres systèmes administratifs beaucoup moins favorables à la centralisation monarchique. Certains len en effet, parmi lesquels les len på agift ou encore les pantelen ${ }^{17}$, ajoutèrent des privilèges particuliers à la rémunération traditionnelle du titulaire.

11 Le pantelen correspondait à la mise en gage d'une terre par la Couronne, en général lorsque celle-ci avait besoin d'obtenir rapidement des liquidités. Comme les bourgeois ne disposaient pas à l'époque de grands capitaux, le roi n'avait pas d'autre solution que de se tourner vers l'aristocratie. L'emprunt public se trouvait ainsi étroitement lié au système des len ${ }^{18}$. Le titulaire d'un pantelen disposait des mêmes pouvoirs administratifs qu'un syslemann et prélevait le remboursement de son prêt sur une part 
des revenus royaux. À l'origine, une convention de gage entre le roi et le seigneur stipulait les conditions d'octroi du pantelen. Mais Birgitta Fritz note qu'en Suède, à partir de 1350 , les précisions concernant la somme annuelle impartie au remboursement du prêt furent négligées. Les pantelen ne furent plus repris du vivant de leurs bénéficiaires et devinrent héréditaires. Certaines sources attestent même de remises en gage directement entre seigneurs ${ }^{19}$. Le système des pantelen fut donc particulièrement favorable à l'aristocratie : non seulement il plaçait la Couronne dans un rapport de dépendance étroit avec ses créanciers, mais il permettait encore au bénéficiaire de s'enrichir personnellement au détriment de la Couronne et de la population. Comme la comptabilité échappait au pouvoir royal, le titulaire d'un pantelen renforçait son monopole sur l'administration du territoire. C'est en Suède que les pantelen constituèrent la forme administrative la plus répandue. Sous le règne $\mathrm{d}$ 'Albert de Mecklembourg (1364-1389), de puissants magnats, à l'instar de Bo Jonsson (Grip) ${ }^{20}$, dominèrent ainsi de grandes portions du territoire du royaume. Au Danemark, pour bénéficier du soutien politique du Conseil du royaume (Rigsråd) au moment de l'élection de son fils Olaf en 1376 et durant la régence, Marguerite fut amenée à céder de vastes domaines à certains conseillers comme Henning Podebusk ou encore l'archevêque de Lund, Niels Jakobsen (Ulfeldt).

Même si les pantelen furent moins courants en Norvège que dans les deux autres royaumes scandinaves, la lettre d'instructions témoigne de plusieurs mises en gage. Le premier créancier, Gaute Eiriksson (Galtung), était un seigneur laïc fortuné descendant d'une des dernières familles baronniales norvégiennes. Il est prévu dans la lettre qu'Erik lui emprunte 100 nobles ${ }^{21}$ et lui laisse en échange le sysle de Skien jusqu'à remboursement de l'emprunt ${ }^{22}$. Il est ensuite question de deux prélats : l'évêque Jakob de Bergen, susceptible d'« échanger avec [Erik] une somme d'argent contre une terre mise à sa disposition ${ }^{23}$ ", et l'archevêque de Nidaros, Eskil, auquel Erik doit donner en len le fylke de Sparbu, ayant autrefois appartenu à son prédécesseur Vinald Henriksson ${ }^{24}$.

13 La lettre de 1405 témoigne bien de la politique d'emprunt de Marguerite. En Norvège, la reine se tourna en priorité vers les hommes d'église, et cela pour deux raisons majeures. La première est que, contrairement à l'aristocratie laïque généralement peu fortunée, les prélats détenaient une part importante des richesses du royaume. La seconde est que les évêques, nommés par provision pontificale sur recommandation expresse de la Couronne auprès du Saint-Siège, étaient des hommes entièrement dévoués à la cause royale. Plus généralement, si ces pantelen furent souvent la réponse immédiate à un besoin aigu d'argent, ils ne furent pas uniquement le résultat d'une crise. Ils constituèrent une forme administrative particulière qui correspondait à un choix politique délibéré de la part du monarque ${ }^{25}$. La dissolution du système des sysler, aussi paradoxal que cela puisse paraître, fut donc autant le fruit d'une politique royale que la conséquence de l'action de certains seigneurs soucieux de défendre leurs intérêts et d'accroître leurs gains par tous les moyens possibles.

Les « aliénations abusives » des officiers

14 Les remontrances que Marguerite adresse à ses officiers par l'intermédiaire d'Erik, ainsi que ses appels répétés à la vigilance, tendraient à prouver que certains ambitieux cherchèrent à s'enrichir au détriment des finances royales et à établir leur monopole sur les terres et les châteaux de la Couronne. Une première réprimande est formulée à l'attention du chevalier Jakob Fastulvsson ${ }^{26}$ : 
Si herr Jakob se présente à Notre fils et lui réclame un nouveau sysle, [Erik] doit lui répondre qu'il doit avant toute chose rendre des comptes pour le sysle qu'il détient à présent et pour tous les autres qu'il a eus jusque-là ${ }^{27}$.

l'administration nordique entre 1367 et $1405^{33}$. Il explique que la validité d'un document dépendait à l'époque de la qualité du support utilisé, aussi bien que de la manière dont le sceau était apposé. Contrairement au papier qui connut une utilisation tardive en Scandinavie ${ }^{34}$, la peau de parchemin, synonyme pour les gens de l'époque d'authenticité et de perpétuité, conférait une plus grande validité aux actes ${ }^{35}$. En outre, un sceau pendant avait plus de valeur qu'un sceau plaqué au dos du document ${ }^{36}$. À la fin du Moyen Âge, le papier fut généralement utilisé pour l'émission de copies et d'actes administratifs. Ces lettres étaient fermées au dos par des sceaux plaqués, comme des lettres closes. La première recommandation de Marguerite, qui consiste à ne pas sceller de lettre sur parchemin avec un sceau pendant, correspondait à une 
tactique héritée de son père Valdemar. Ce dernier évitait en effet, dans la mesure du possible, de sceller les documents officiels susceptibles de limiter sa liberté d'action ${ }^{37}$. La seconde recommandation, qui préconise de sceller les lettres sur papier au dos à l'aide d'un sceau plaqué, est plus difficile à interpréter. Marguerite se contente peutêtre de dispenser quelques règles élémentaires concernant l'usage d'un support nouveau. Elle introduit aussi, consciemment ou pas, une distinction entre l'acte à caractère politique, qui nécessitait un engagement important du pouvoir royal, et l'acte administratif, qui s'inscrivait déjà dans une forme de routine bureaucratique. Peut-être s'agit-il enfin d'une mise en garde contre d'éventuels risques de falsification documentaire : le papier n'était pas le support idéal pour recevoir un sceau, dans la mesure où un sceau fixé à l'œillet d'une lettre sur papier pouvait facilement être coupé ou arraché.

Dans l'acte de couronnement du 13 juillet 1397, les représentants des trois royaumes promirent de laisser à la complète disposition de Marguerite et d'Erik les châteaux, forteresses, terres et len qui leur avaient été confiés ou leur seraient confiés à l'avenir ${ }^{38}$. Autrement dit, les seigneurs s'engageaient à laisser les monarques libres de nommer les officiers qu'ils souhaitaient aux postes qu'ils voulaient. En outre, les fondements d'un pouvoir royal délégué par Dieu furent clairement définis ${ }^{39}$. Dans la lettre de 1405, Marguerite rappelle encore une fois avec insistance que le roi tient son pouvoir de Dieu et agit en son nom. À plusieurs reprises, par l'intermédiaire d'Erik, elle somme ses officiers de « rendre au roi ce qui lui revient, quand celui-ci le leur réclame au nom de Dieu ${ }^{40}$. Un seigneur risquait de s'opposer à la nomination de son successeur. Il s'agissait du chevalier Ogmund Berdorsson (Bolt), capitaine de la forteresse d'Akershus depuis plus de quinze ans :

Si herr Ogmund l'invite à reprendre la forteresse, qu'[Erik] la prenne au nom de Dieu. S'il ne l'y invite pas, qu'[Erik] la lui réclame au nom de Dieu et la lui prenne ${ }^{41}$.

La nomination d'un officier s'accompagnait obligatoirement d'un échange de lettres. L'autorité que le roi conférait à cet homme et les conditions de l'octroi du sysle étaient stipulées dans la syslebrev. De son côté, le syslemann éditait une contre-lettre (motsbrev) dans laquelle il prêtait le serment évoqué précédemment ${ }^{42}$. Ce rituel, orchestré par le roi, était un moyen pour ce dernier de réaffirmer aussi bien sa souveraineté sur les terres de la Couronne que sa prérogative en matière de nomination. À en croire le passage suivant, il est probable que certains officiers n'aient pas respecté la procédure :

Si quelqu'un en Norvège réclame [à Erik] des lettres pour des châteaux, qui reconnaîtraient qu'il a reçu ces châteaux d'un tiers, qu'[Erik] se garde bien de délivrer de telles lettres. Car il est de notoriété publique que [le roi] les a déjà reçus de cette personne ${ }^{43}$.

Le passage est un peu obscur et il convient de rester prudent dans son interprétation. Je pense que Marguerite dénonce ici le transfert de sysler d'un détenteur à un autre, sans qu'elle-même ou Erik en aient été avisés. En matière de nomination d'officiers, le pouvoir royal avait manifestement du mal à maitriser les diverses formes de cooptation et parfois même d'opposition. Un syslemann semble en effet avoir été contesté dans l'exercice de ses fonctions et Marguerite met en garde Erik contre l'éventualité d'une usurpation par la violence :

De plus, qu'[Erik] ne laisse personne avoir le pouvoir de destituer celui-ci ou de prendre le len dont il est actuellement titulaire, à l'exception de Dieu, de lui-même et de Nous ; car il en est certains qui lui veulent du mal parce qu'il est étranger et maintient les droits de la Couronne ${ }^{44}$. 
est difficile d'établir avec certitude l'identité du syslemann dont il est question dans cet extrait. Michael Linton pense qu'il pourrait s'agir de Claus Grubendal, qu'on retrouve à la tête de la forteresse de Bohus deux ans plus tard ${ }^{45}$. Issu d'une famille originaire du Holstein, dont une branche avait émigré au Danemark au XIII ${ }^{e}$ siècle, cet " étranger » était alors membre du Conseil du royaume danois. La lettre d'Union du 20 juillet 1397 stipulait que les royaumes devaient être gouvernés d'après leur loi respective ${ }^{46}$. En Suède, la loi du royaume, rédigée dans les années 1350 , prévoyait que seuls les autochtones étaient en droit de détenir les châteaux et les slottslen de leur royaume d'origine ${ }^{47}$. Or, ni la loi du royaume ni la lettre d'Union ne dissuadèrent Marguerite et Erik de nommer à des postes de l'administration suédoise des officiers danois et allemands ${ }^{48}$. Ces nominations furent par ailleurs fortement décriées par l'aristocratie suédoise en révolte dans les années 1430.

Dans quelle mesure Erik eut-il à user des réprimandes dictées par la reine mère ? Les sources postérieures ne disent rien des conditions dans lesquelles le chevalier Ogmund Berdorsson (Bolt) céda sa place à Eindride Erlendsson (Losna) à la tête de la forteresse d'Akershus en 1405. En outre, elles n'attestent d'aucun soulèvement en Norvège avant 1436. Je pense pour ma part que si Marguerite fit preuve d'autant d'autorité dans son royaume héréditaire, c'est qu'elle disposait là des moyens de l'exercer. En Norvège en effet, ses positions étaient mieux assises qu'ailleurs. L'aristocratie norvégienne, économiquement moins puissante, fut certainement moins turbulente que ses voisines. Cet état de fait, qui présente une certaine distorsion avec ce que suggèrent les instructions, conduit à s'interroger sur la nature véritable de cette lettre. Parce que le terrain s'y prêtait sans doute mieux qu'ailleurs, les affaires norvégiennes étaient l'occasion d'exprimer un véritable programme de gouvernement.

Stratégies royales et consolidation du système administratif des sysler

compter de son élection dans les trois royaumes comme " maîtresse toute-puissante et seigneur légitime ${ }^{49} "$, Marguerite eut besoin d'un peu moins d'une quinzaine d'années pour poser les bases d'un pouvoir monarchique fort ${ }^{50}$. Elle œuvra avec une grande habileté pour restaurer le domaine royal. Elle mit en place une forme d'autocratie politique en s'abstenant de pourvoir les postes laissés vacants par les grands officiers du royaume et en transférant leurs compétences à des personnes entièrement dévouées à sa cause ${ }^{51}$.

Pour réaffirmer sa souveraineté sur le territoire, la reine devait au préalable affermir le contrôle de la Couronne sur les pantelen. Elle profita du couronnement d'Erik au Danemark et en Suède pour reprendre possession des terres domaniales tombées aux mains de l'aristocratie du royaume ${ }^{52}$. Officiellement entamée en 1396, cette restitution se poursuivit durant tout le premier quart $\mathrm{du} \mathrm{xv}^{\mathrm{e}}$ siècle. Elle toucha en particulier la Suède et le Danemark où le nombre des pantelen était élevé. La Norvège fut également concernée : en 1401, Gaute Eiriksson (Galtung) dut restituer à la Couronne le sysle de Skien dont il était titulaire depuis $1386^{53}$ et reçut en compensation le sysle de Nordmøre ${ }^{54}$. Marguerite explique à Erik comment procéder concrètement pour poursuivre cette réforme. Il s'agissait tout d'abord de récupérer les anciennes lettres données aux seigneurs par les souverains précédents ou elle-même. L'évêque de Bergen fut chargé de rassembler un certain nombre de ces lettres:

Nous lui avons ordonné de reprendre toutes nos lettres de protection (vernebrev), les lettres d'exemption (frälsebrev) et celles accordées pour les bateaux en partance 
pour l'Islande. Nous savons qu'il s'est exécuté ; et quiconque voudrait récupérer ces lettres doit les faire renouveler par Notre fils ou Nous-même ${ }^{55}$.

La loi voulait en effet que le souverain, à son avènement, confirmât les décisions prises par ses prédécesseurs en matière d'octroi de terre, de privilège ou encore d'exemption. C'était, semble-t-il, la première fois qu'Erik se rendait en Norvège depuis la proclamation de sa majorité : il était probable qu'à cette occasion les seigneurs ne manqueraient pas de faire valoir leurs droits sur les terres dont ils avaient été jusque-là titulaires. Marguerite conseille donc à Erik de retarder au maximum la confirmation ou le renouvellement de ces lettres :

Si quelqu'un en Norvège se présente à lui avec d'anciennes lettres que le roi Magnus, le roi Håkon, Nous-même ou d'autres ont délivrées concernant des biens, des len ou des exemptions (...), et en réclame le renouvellement, [Erik] doit répondre qu'il ne sait pas ce qui a été donné avant que Nous arrivions ${ }^{56}$.

Marguerite fixe enfin de nouvelles conditions d'octroi aux len ainsi rattachés au domaine royal. Ces conditions visaient naturellement à limiter l'autorité des futurs bénéficiaires. Pour éviter qu'ils ne devinssent héréditaires, les sysler furent dès lors confiés pour une durée laissée à la libre appréciation du roi. L'archevêque Vinald Henriksson avait été titulaire sa vie durant du len de Sparbu. Son successeur Eskil devait en disposer « aussi longtemps que sa Grâce le dirait ${ }^{57}$ ». D'autre part, pour éviter que les officiers n'accrussent leur rémunération au détriment des revenus de la Couronne, la reine renforça la comptabilité sur ses terres. Deux solutions s'offraient à elle : soit elle transformait les pantelen en len på regnskap ${ }^{58}$ (Gaute Eiriksson, détenteur du pantelen de Skien, reçut Nordmøre på regnskap), soit elle changeait les conditions d'octroi des pantelen. Ainsi, Eskil reçut le pantelen de Sparbu «en pleine comptabilité, avec amortissement du prêt annuel, jusqu'à remboursement de la somme ${ }^{59}$ ».

La récente majorité du roi avait entraîné un remaniement au sein du personnel administratif norvégien. La lettre de 1405 témoigne de plusieurs nominations, dont celle d'Eindride Erlendsson (Losna) au poste de capitaine de la forteresse d'Akershus ${ }^{60}$. Cet homme était membre d'une famille de serviteurs royaux, fidèles à la dynastie des Folkungar depuis trois générations. Son père, Erlend Filipusson (Losna), avait été fehirde de Bergen entre 1375 et 1390 et membre du Riksråd norvégien au moment de la minorité d'Olaf Håkonsson. Preuve de la faveur royale accordée à la famille Losna, la sœur d'Eindride, Sigrid Erlendsdatter, épousa un membre de la famille royale, Håkon Sigurdsson de Giske. Eindride devait avoir une trentaine d'années environ ${ }^{61}$ et faisait donc partie de la nouvelle génération de riksråder. Dans la lettre de 1405, il doit seconder Erik durant son voyage en Norvège et l'escorter impérativement sur le trajet qui sépare Akershus de Tønsberg:

$\mathrm{Qu}^{\prime}$ (Erik) prenne herr Eindride avec lui (...) car il doit toujours avoir quelqu'un à ses côtés (...). [Erik] et herr Eindride doivent nommer à Akershus un Norvégien de leur choix pour le temps qu'ils passeront à Tønsberg. Et herr Eindride doit impérativement rester avec (Notre fils) ${ }^{62}$.

En confiant à Eindride Erlendsson la forteresse d'Akershus, et un peu plus tard celle de Tønsberg, Marguerite laissait aux mains d'un seul et même homme deux des plus importantes régions administratives norvégiennes. Le second promu signalé dans la lettre est un étranger : il s'agit de Claus Grubendal, homme sans pouvoir local, et de ce fait extrêmement dépendant du prestige que lui conférait le service du roi. Nommé à Bohus, il se vit confier un poste à haute responsabilité dans la mesure où cette forteresse, située à la frontière dano-suédoise et en bordure du Sund, était un point 
stratégique économique et militaire de première importance. Le choix de nommer des seigneurs étrangers à la tête de cette région se confirma les années suivantes ${ }^{63}$.

Eindride Erlendsson et Claus Grubendal ne furent pas seulement nommés à des postes importants de l'administration locale. La lettre de 1405 atteste aussi de leur implication dans l'administration centrale du royaume. En 1400, Eindride fut nommé au poste de kjøkemester ${ }^{64}$ et Claus remplit probablement la fonction de chancelier. À la fin du $\mathrm{XIV}^{\mathrm{e}}$ siècle, les deux derniers grands officiers du royaume qui subsistaient encore en Norvège étaient le sénéchal (drost ${ }^{65}$ ) et le chancelier. En 1388, le drost Ogmund Finsson til Hestbø mourut et la reine négligea de pourvoir le poste après lui. Le chancelier continua d'exercer sa fonction, bien qu'il fût privé d'une grande partie de ses compétences, en particulier de celle de garde des sceaux. En 1405, Marguerite refuse de restituer aux seigneurs norvégiens le sceau du royaume transféré au Danemark en $1398^{66}$ :

Si les Norvégiens veulent reprendre possession du sceau du royaume (...), [Erik] doit leur dire qu'il ne le leur rendra pas avant de s'être entretenu avec le Riksråd au sujet de la personne qu'il entend nommer (au poste de chancelier); et si on lui réplique que (cette personne) ne peut pas être à la fois fogde et chancelier, [Erik] doit répondre qu'il s'enquerra lui-même de savoir comment (cet homme) entend s'acquitter de sa tâche (...); car Nous pensons vraiment qu'il est en mesure de remplir les deux fonctions à la fois ${ }^{67}$.

L'office de chancelier avait été jusque-là l'attribution du prévôt de la chapelle royale d'Oslo (prost) : nommer un laïc était une entorse à la tradition, que Michael Linton interprète comme la preuve de la mise en place par Marguerite et Erik d'une hégémonie des fogder ${ }^{68}$.

L'effort de centralisation était manifeste. La reine supprima les intermédiaires ou les institutions trop indépendantes susceptibles de gêner la mise en place d'un pouvoir monarchique fort. Elle plaça ses créatures : localement, en nommant à la tête des sysler des fonctionnaires qui dépendaient directement d'elle, et au sein du gouvernement central, en remplaçant les anciens officiers du royaume (riksembetsmenn), qui avaient acquis une certaine autonomie pendant la régence et les absences chroniques du roi, par des officiers de cour (hoffembetsmenn) sur lesquels elle pouvait exercer une plus étroite surveillance et dont Eindride Erlendsson et Claus Grubendal sont les meilleurs représentants.

Ces nominations se firent au détriment de plusieurs chevaliers puissants et influents, à l'instar de Jon Reidarsson (Darre), Ogmund Berdorsson (Bolt) ou encore Gaute Eiriksson (Galtung). Relativement âgés, ces chevaliers avaient profité d'une longue carrière au service du roi pour accumuler richesses et prestige. Ils avaient construit un réseau de sociabilité important au-delà même des frontières du royaume et formaient un groupe soudé d'individus avec lequel la Couronne devait composer. Marguerite recommande donc à Erik d'éviter les impairs et propos fâcheux, de ménager les susceptibilités en acceptant les invitations à dîner, les cadeaux et en dispensant flatteries et promesses ${ }^{69}$. Il aurait été maladroit de priver ces hommes de leurs offices sans prévoir de compensation: Gaute Eiriksson reçut le sysle de Nordmøre contre celui de Skien et Jon Darre le sysle de Lista en l'échange de celui de Tønsberg ${ }^{70}$. Marguerite profita de ces mutations pour troquer des slottslen contre des sysler périphériques et sans château. Toutefois, la compensation devait être suffisamment attractive pour que l'officier muté ne se sentît pas lésé. Gaute Eiriksson (Galtung), dont la plupart des propriétés se situaient dans le quart sud-est du royaume (en Telemark, Borgarsysle et Vestfold), fut 
envoyé dans le nord du royaume. Malgré l'éloignement, il n'est pas impossible que Gaute ait trouvé son compte dans l'échange, dans la mesure où il avait des affaires sur les îles Féroé ${ }^{71}$.

Quelles raisons poussèrent Marguerite à écarter des plus hauts postes de l'administration locale ces hommes, serviteurs fidèles de Håkon VI et d'Olaf, qui en 1388 la désignèrent comme régente à vie du royaume de Norvège, acclamèrent Erik de Poméranie comme successeur au trône, le couronnèrent roi de Norvège en 1392 et de l'Union cinq ans plus tard? Leur appartenance au Riksråd n'est certainement pas étrangère à cet ostracisme. Dans la lettre, le Conseil du royaume apparaît comme un cadre légal avec lequel le monarque devait composer :

Concernant les riksråder (...), il doit convoquer tantôt les uns tantôt les autres ; qu'il les flatte du mieux qu'il peut; et s'il a une affaire extraordinaire à traiter, qu'il les consulte à ce sujet et leur demande leur avis; et s'il doit prononcer une sentence à quelque jugement, qu'il dise ce qui est bon et raisonnable, d'après les prescriptions de la loi ${ }^{72}$.

Pour imposer ses vues politiques et contourner les résistances des membres les plus influents, Marguerite sut habilement jouer sur la composition du Conseil en variant le recrutement. Elle chercha à limiter le pouvoir de décision de l'assemblée en la confortant dans son rôle consultatif originel. Plusieurs historiens, parmi lesquels Ole J. Benedictow, estiment que la politique de Marguerite tendit à éliminer le Riksråd comme force politique ${ }^{73}$. L'assemblée cessa de se réunir en tant que telle à partir de 1392 et les conseillers norvégiens furent noyés dans la masse des riksråder scandinaves lors des réunions communes organisées par les monarques à partir de 1395. En outre, le Conseil perdit une grande partie de son autonomie avec le transfert du sceau norvégien au Danemark en $1398^{74}$. Le retrait des slottslen des mains des membres du Riksråd aurait participé de cette politique d'affaiblissement du Conseil en tant qu'organe de gouvernement. Le contrôle des len, instruments militaires et économiques, était une des conditions permettant à l'aristocratie de maintenir sa position dans la société. Jakob T. Larsen, dans son ouvrage consacré au Riksråd norvégien entre 1434 et 1450, montre que le Conseil chercha constamment à contrôler les nominations pour réserver l'attribution des len à ses membres. De leur côté, les monarques mirent les len au service de leurs intérêts politiques; par leur biais, ils diminuèrent ou augmentèrent le poids de leur Conseil au gré des besoins. Ainsi, dans la période troublée des années 1430, Erik tint les titulaires des len en dehors du Riksråd. Au contraire, le roi Christophe, en pleine crise avec les Hanséates, accorda des len à ses conseillers pour renforcer le Riksråd dont l'appui politique lui était devenu nécessaire ${ }^{75}$.

Dans sa lettre, la reine Marguerite définit les principes d'un pouvoir monarchique fort, fondé sur le contrôle des terres, par la réaffirmation du principe d'inaliénabilité du domaine royal, et sur le contrôle des hommes, par la soumission au prince du personnel administratif. En cela, elle s'inscrit pleinement dans la lignée des souverains continentaux qui cherchèrent à renforcer leur position et à se libérer de la dépendance des grands seigneurs ${ }^{76}$.

La mise en œuvre de ce programme de réformes eut bien sûr ses limites. Les accusations portées par l'historien Ericus Olai témoignent, quelques décennies plus tard, des réactions que la politique de Marguerite suscita auprès de la puissante aristocratie suédoise :

À son avènement, elle (...) délivra beaucoup de longues et belles lettres où elle promit que les châteaux suédois ne seraient confiés qu'à des autochtones. Elle n'en 
n'attribua pas moins ces châteaux à des Danois et des Allemands et nomma des officiers et des fogder, les plus vils et plus rusés qui fussent (tout comme elle l'était), des extorqueurs de fonds de la pire espèce, de sorte qu'ils dépecèrent, arrachèrent les entrailles et laissèrent le pauvre royaume des Goths exsangue ${ }^{77}$.

En Norvège, Marguerite rencontra moins de résistances. Premièrement, le royaume était héréditaire et portait donc en soi les bases d'une forte légitimation monarchique. Ensuite, le système des sysler était moins perverti que dans les deux autres royaumes. Enfin, l'aristocratie norvégienne, durement frappée par la peste des années 1350 et par la crise agraire qui s'ensuivit, était numériquement et économiquement plus faible que ses voisines, ses réseaux d'alliance et de clientèle moins efficaces. La position sociale des seigneurs norvégiens dépendait donc pour l'essentiel de leur service et de leur attachement au roi.

Les affaires norvégiennes, dans la mesure où la reine en avait le contrôle, offrirent un cadre idéal à la formation princière. Cette lettre n'est pas sans rappeler les manuels de bon gouvernement, écrits et inspirés par des rois soucieux de tracer pour leur successeur le comportement à suivre de manière à le conduire au faîte de ses responsabilités ${ }^{78}$. Il est bien possible que la reine se soit inspirée, soit directement, soit indirectement, dans les principes qui sous-tendent les instructions, du fameux Konungs skuggsiá (Miroir du roi) ${ }^{79}$, écrit sans doute vers le milieu du XIII ${ }^{\mathrm{e}}$ siècle en Norvège. L'auteur anonyme s'y révèle un ferme partisan d'un pouvoir royal fort et de l'inaliénabilité des biens de la Couronne ${ }^{80}$. Cependant, les vertus chrétiennes, exaltées par les miroirs royaux des siècles précédents, passent ici au second plan ${ }^{81}$ : la lettre de 1405 est avant tout un guide concret de gouvernement, dont le pragmatisme politique témoigne de la lente transition d'une monarchie féodale à une monarchie administrative au centre de laquelle le prince affiche un pouvoir toujours plus affirmé ${ }^{82}$.

\section{NOTES}

1. C. DE PIZAN, Le chemin de Longue étude (édition critique du ms. Harley 4431 et traduction par A. Tarnowsky), Paris, 2000, p. 225-226.

2. La lettre aurait été rédigée entre le 21 janvier et le 15 février 1405 . Cf. M. LiNTON, Drottning Margareta, fullmäktiga fru och rätt husbonde, Studia historica gothoburgensia, XII, 1971, p. 286. Cf. Norske Middelalder Dokumenter, S. BAGGE, K. HELLE et S. HoLSTAD SMEDSDAL éd., Oslo, 1973 (désormais abrégé en : NMD), $\mathrm{n}^{\circ} 105, \S 20$. Nous avons conservé l'original sur papier et une copie des années 1750 . Le texte est lacunaire et comporte des passages assez obscurs. Cette lettre en ancien danois comporte quelques termes suédois et norvégiens.

3. NMD, $\mathrm{n}^{\circ} 105, \S 41$.

4. Tout au long de l'article, les termes scandinaves seront cités, pour la plupart, dans leur forme norvégienne.

5. B. GuENÉE, L'Occident aux XIV et XV siècles. Les États, Paris, 1971, p. 164. 
6. P. S. ANDERSEN, « Syssel », dans Kulturhistorisk Leksikon för Nordisk Middelalder (désormais abrégé en : KLNM), XVII, p. 646.

7. La royauté norvégienne contrôlait alors un vaste territoire allant de l'actuelle région suédoise de Bohuslän jusqu'au Finnmark, en passant par les régions de Herjedalen et de Jemtland le long de la frontière suédoise. Elle étendait également sa domination sur les îles Shetland, Orcades et Féroé, ainsi que sur l'Islande et le Groenland.

8. P. S. ANDERSEN, « Sysselmann », dans KLNM, XVII, p. 651-656.

9. G. A. BLOM, « Fehirde », dans KLNM, IV, p. 211.

10. B. FRITZ, Hus, land och län. Förvaltningen i Sverige 1250-1434, Stockholm, 1972.

11. Il s'agit probablement de Tideke Henriksson (Vistenaker).

12. Un skipreide était une entité administrative située sur le littoral, dont les habitants avaient pour obligation de verser le leidang, contribution financière destinée à

l'armement de navires en temps de guerre. Cf. H. BJøRKVIK, "Skipreide », dans KLNM, XV, p. 546-551.

13. Au sujet de la propriété de l'office, voir l'article de R. DESCIMON, « Les Élites du pouvoir et le prince : l'État comme entreprise ", dans Les Élites du pouvoir et la construction de l'État en Europe. Les origines de l'État moderne en Europe, W. REINHARD dir., Paris, 1996, p. 133-162 (en particulier les pages 147 à 153).

14. Norsk historie, I. 750-1537, O. G. MosenG, E. OPSAHL et alii éd., Oslo, 1999, p. 191.

15. P. S. ANDERSEN, « Syssel », loc. cit., p. 647-648; Norsk historie, op. cit., p. 191.

16. E. OPSAHL, « Bastard Feodalism or Sub-vassality in Medieval Norway? ", dans Collegium Medievale, vol. 4, Oslo, 1991-1992, p. 179.

17. J. RosÉN, « Län », dans KLNM, XI, p. 106-107. Len på agift : fief concédé moyennant le versement d'une redevance annuelle. Pantelen : fief laissé en gage pour dette. Les revenus du fief permettaient à son titulaire de recouvrer la somme empruntée.

18. Cf. B. POULSEN, « Royaumes à la périphérie de l'Europe : la Scandinavie médiévale et moderne ", dans Systèmes économiques et finances publiques. Les origines de l'État moderne en Europe, Paris, 1996, p. 101.

19. B. FRITZ, op. cit., p. 94-107.

20. Les historiens scandinaves présentent l'identité des individus de la manière suivante : ils donnent le prénom et le patronyme rapportés par les sources et précisent le nom de famille, généralement utilisé plus tardivement par les membres du lignage, entre parenthèses.

21. La valeur du noble, monnaie d'or anglaise, correspondait en 1412 à celle d'un double florin (florentin). Cf. P. SPUFFORD, Money and its use in Medieval Europe, Cambridge, 1993 , p. 282 et 320.

22. NMD, $\mathrm{n}^{\circ} 105, \S 30$.

23. Ibid., § 31 .

24. Ibid., § 32. Le fylke était une entité administrative issue d'un maillage administratif ancien, caractéristique des régions de Vestlandet et de Trøndelag. Cf. S. BAUGE, « Fylke », dans KLNM, V, p. 546-551.

25. Voir à ce sujet, à titre comparatif, J. MORSEL, "À quoi sert le service de l'État ? ", dans Les Serviteurs de l'État au Moyen Âge, XXIX Congrès de la SHMESP, Pau, mai 1998, Paris, 1999, p. 238.

26. Cf. M. LINTON, op. cit., p. 294.

27. NMD, $\mathrm{n}^{\circ} 105, \S 25$.

28. Ibid., § 24 . 
29. Ibid., § 13 .

30. Ibid., § 37 .

31. A. E. CHRISTENSEN, Kalmarunionen og nordisk politik 1319-1439, Copenhague, 1980,

p. 177.

32. $N M D, \mathrm{n}^{\circ} 105, \S 28$.

33. M. LiNTON, op. cit., p. 302-312.

34. Le nonce pontifical Pierre Gervais raconte que, lors de son séjour en Scanie dans les années 1330, il fut contraint d'acheter des peaux de parchemin, étant donnée l'absence de papier dans le royaume. Cf. É. MORNET, «Per niues de nocte : un nonce pontifical dans les royaumes nordiques au XIV ${ }^{\mathrm{e}}$ siècle ", dans Milieux naturels, espaces sociaux. Études offertes à Robert Delort, Paris, 1997, p. 592, note 5.

35. O. Guyotjeannin, J. Pycke et B.-M. Tock, Diplomatique médiévale, Turnhout, 1993, p. 64-65.

36. M. LiNTON, op. cit., p. 308.

37. Le roi danois refusa par exemple de sceller avec le grand sceau du royaume le traité de paix qu'il passa avec les villes marchandes de la Hanse à Stralsund en 1370. Cf. M. LinTON, op. cit., p. 305.

38. Sverges Traktater med Främmande Magter, O. S. RYDBERG et alii éd., Stockholm, 1877-1934, nº 423 (désormais abrégé en : ST).

39. Cf. E. LÖNNROTH, Sverige och Kalmarunionen 1397-1457, Göteborg, 1934, p. 46.

40. $N M D, \mathrm{n}^{\circ} 105, \S 53$.

41. Ibid., $\S 9$.

42. L. HAMRE, « Motsbrev », KLNM, XI, p. 709-710. Olivier Mattéoni rappelle l'importance du serment de nomination dans la société politique de la fin du Moyen Âge et insiste sur la solennité qui entourait sa prestation. Cf. O. MATTÉONI, Servir le prince. Les officiers des ducs de Bourbon à la fin du Moyen Âge (1356-1523), Paris, 1998, p. 251-257.

43. NMD, $\mathrm{n}^{\circ} 105, \S 53$.

44. Ibid., § 21 .

45. Claus Grubendal scella un acte daté du 3 mars 1407 en qualité d'høvedsmann de Bohus (cf. Diplomatarium Danicum, 4-11, n 45). Nous n'avons pas conservé sa lettre de nomination et peut-être était-il déjà en poste en 1405. Mais il pourrait s'agir également de son fils, Fikke Grubendal, titulaire du syssel de Skiksvik et du château de Dyngehus, dont la lettre d'octroi du 21 janvier 1405 fut scellée par plusieurs des officiers envers lesquels la reine témoigne une certaine méfiance en 1405, parmi lesquels Ogmund Berdorsson (Bolt) et Jon Reidarsson (Darre) (cf. Diplomatarium Norvegicum [DN], 16, $\left.n^{\circ} 50\right)$. Un indice joue néanmoins en la faveur de Claus : il est possible que Marguerite omette de mentionner au paragraphe 21 le nom de l'officier, dans la mesure où elle y fait déjà allusion au paragraphe précédent. Le paragraphe 20 signale en effet que l'office de chancelier serait désormais attribué à un fogde. Or Claus Grubendal a vraisemblablement rempli la fonction de chancelier les années suivantes. $\mathrm{Cf}$. M. LINTON, op. cit., p. 293.

46. ST, II, p. 563-567.

47. Cf. Magnus Erikssons Landslag, Rättshistoriskt bibliotek, 1-7, Å. HOLMBÄCK et E. WESSEN éd., Lund, 1962, p. 5.

48. J. E. OLESEN, « The governmental System in the Union of Kalmar, 1389-1439 », dans Studien zur Geschichte des Ostseeraumes, I, T. RIIs éd., Odense, 1995, p. 59.

49. « Fullmäktig fru och rätt husbonde ». 
50. A. E. Christensen, op. cit., p. 229.

51. Ibid., p. 236-238.

52. L'acte principal de cette réforme fut le décret rédigé en septembre 1396 à

Nyköping. Cf. ST, II. Bihang, p. 655-664.

53. E. HAUG, Margrete, den siste dronning i Sverreaetten, Nordens fullmektige frue og rette husbonde, Gjøvik, 2000, p. 283.

54. $D N, 1, \mathrm{n}^{\circ} 575$.

55. $N M D, \mathrm{n}^{\circ} 105, \S 35$.

56. Ibid., § 35 .

57. Ibid., § 32 .

58. Littéralement, « fief en compte »; voir supra, p. 105-106. Voir J. E. OLESEN, « Middelalderen till 1536 : fra rejsekongedømme til administrationscentrum », dans Dansk Forvaltningshistorie, I. Stat, Forvaltning og Samfund fra Middelalderen til 1901, Copenhague, 2000, p. 28-29.

59. « (...) han lade hanom thet sammae laen vnder fullaen greyn oc raekning hwort aar aff atsla, til thes han hauer wpboret swo meghit, som han ther vpa wt laener ». Cf. NMD, $\mathrm{n}^{\mathrm{o}} 105, \S 32$.

60. Ibid., § 9 .

61. Eindride apparait pour la première fois dans les sources en 1384 . Il décéda en 1440.

62. $N M D, \mathrm{n}^{\circ} 105, \S 14$.

63. Trois Allemands s'y succédèrent jusqu'en 1440 : Claus Grubendal (av. 1407-1415) ; Hans Kröpelin (1419-1423) et Albert Bydelsbach (1423-1440). Cf. O. J. BENEDICTOW, Den nordiske adel $i$ Senmiddelalderen. Struktur, funktioner og internordiske relationer. Rapporter til det nordiske historikermøde i København 9-12 august 1971, Copenhague, 1971, p. 29-30.

64. $N M D, \mathrm{n}^{\circ} 105, \S 9$. Au XII ${ }^{\mathrm{e}}$ siècle, le kjøkemester était un officier chargé de l'approvisionnement de la cour royale. Disparu au XIII ${ }^{\mathrm{e}}$ siècle, le terme réapparaît sous le règne de Marguerite. L'officier reprend vraisemblablement les fonctions politiques du drost. Cf. E. HAUG, op. cit., p. 262-263.

65. Le drost (en latin dapifer) fonctionnait comme un vice-roi au moment des régences. Il se trouvait alors à la tête du Conseil royal.

66. Le sceau du royaume ne fut plus jamais utilisé comme sceau royal après 1398 . Il fut à nouveau utilisé à partir de 1442 comme sceau du retterting (tribunal royal). Cf.

J. E. OLESEN, « Union og kancelli i det 15. århundrede », dans Ny väg till medeltidsbreven, Stockholm, 2002, p. 56.

67. NMD, $\mathrm{n}^{\circ} 105, \S 20$.

68. M. LINTON, op. cit., p. 293.

69. $N M D, \mathrm{n}^{\circ} 105, \S 11$.

70. Ibid., § 36 .

71. Cf. $D N, 2, \mathrm{n}^{\circ} 559$.

72. NMD, $\mathrm{n}^{\circ} 105, \S 39$.

73. O. J. BENEDICTOW, op. cit., p. 31.

74. M. LINTON, op. cit., p. 293.

75. J. T. LARSEN, Det norske Riksråd 1434-1450. Sammensetning og organisasjon, Bergen, 1970, p. 64-83.

76. B. GUENÉE, op. cit., p. 79 : «Et, en deçà des frontières, le prince ressaisit peu à peu les vieux droits régaliens, impose de mieux en mieux sa justice et sa fiscalité, grâce à des agents de plus en plus nombreux inspirés et contrôlés, de la capitale, par des services 
de plus en plus étoffés. Ainsi se développe après l'État féodal, grâce à l'action de l'administration princière, l'État territorial ». Les souverains ne cherchèrent pas seulement à se libérer de la dépendance fiscale des grands seigneurs. Richard W. Kaeuper montre par exemple les efforts des souverains anglais et français de la fin du Moyen Âge pour obtenir le monopole de la violence légitime. L'historien souligne entre autres l'importance de l'œuvre réformatrice de Philippe V, qui participa à l'élargissement du rôle de la Couronne dans le fonctionnement de la justice et le maintien de l'ordre public. Cf. K. W. KAEUPER, Guerre, justice et ordre public. La France et l'Angleterre à la fin du Moyen Âge, traduction par N. et J.-P. GENET, Paris, 1994.

77. M. LiNTON, op. cit., p. 13. Extrait tiré des Scriptores Rerum Suecicarum Medii Aevi, II, Uppsala, 1828, Livre V, p. 120.

78. À titre d'exemples, voir les Enseignements de saint Louis à son fils Philippe, ou encore le Rosier des guerres commandé par Louis XI à l'attention du dauphin Charles. Cf. J. LE GOFF, Saint Louis, Paris, 1996, p. 418-431 ; J. KRYNEN, L'empire du roi. Idées et croyances politiques en France, XIII'-XVe siècle, Paris, 1993, p. 225-239.

79. Konungs skuggsiá, L. HOLM-OLSEN éd., Oslo, 1945 (reprint, 1982). Voir S. BAGGE, The Political Thought of the King's Mirror, Odense, 1987.

80. Voir S. BAGGE, op. cit., p. 185-186, p. 194 et suiv.

81. Erik doit gouverner avec « sagesse, bonté, équité et tolérance ». Voir NMD, $\mathrm{n}^{\circ} 105$, $\S 41-42$.

82. Selon Jacques Krynen, les miroirs royaux de la fin du Moyen Âge attestent d'une technicité croissante de la mission royale : « Aux XIV et Xve siècles, les miroirs du Prince vont refléter l'accroissement contemporain des tâches du souverain et la complexité grandissante de la fonction proprement dirigeante. Si le métier de roi suppose toujours une panoplie de belles vertus, s'il faut se gouverner soi-même pour gouverner les autres, force est désormais d'expliquer au prince, avec toujours plus de précision, ce que gouverner les autres implique de concret ». Voir J. KRYNEN, op. cit., p. 187-188; J. BLANCHARd et J.-C. MÜHLETHALER, Écriture et pouvoir à l'aube des temps modernes, Paris, 2002, p. 29 et suiv.

\section{RÉSUMÉS}

La lettre que la reine Marguerite adressa en 1405 à son petit-neveu Erik de Poméranie est conçue comme un dialogue entre le jeune roi et les officiers norvégiens. Elle donne un éclairage intéressant sur les relations de pouvoir qui existaient entre la Couronne et les officiers locaux. Elle montre d'une part les résistances de l'aristocratie norvégienne cherchant en priorité à sauvegarder leurs propres intérêts et d'autre part les réformes que la monarchie s'efforçait de promouvoir pour combattre les usurpations de terres et de revenus par cette aristocratie et réaffirmer la souveraineté royale. Cette lettre, qui peut s'apparenter aux manuels de «bon gouvernement ", est représentative des stratégies politiques continûment poursuivies par la reine, caractérisées par une volonté de forte centralisation du pouvoir monarchique et par une forme d'autocratie. 
Queen Margaret and her officials, and the letter she adressed to Erik of Pomerania in 1405. In the letter Margaret adressed to her grand-nephew Erik of Pomerania in 1405, the Queen envisaged a dialogue between the young King and the Norwegian officials. This exchange gives an interesting glimpse into the relations of power which existed between the Crown and its local servants. On one hand, it is significant of the resistances build up by some aristocrats acting first of all to preserve their own interests, and on the other hand, it describes the reforms that the monarchy established in order to fight against the usurpation of land and revenue by the aristocracy and to reaffirm its sovereignity on the Kingdom. This letter, which could be linked to the manuals of "good government", is representative of Margaret's continuous political policies, characterised by strong monarchic centralisation and a form of autocracy, the basis of an absolute power.

\section{INDEX}

Mots-clés : souveraineté, centralisation monarchique, domaine royal, aliénations abusives, personnel politique

Keywords : sovereignty, centralized monarchy, royal estate, illegal appropriations, political staff

\section{AUTEUR}

\section{RAPHAËLLE SCHOTT}

Université Paris I Panthéon-Sorbonne, LAMOP, 17, rue de la Sorbonne, 75005 Paris Göteborgs Universitet, Historiska Institutionen, Box 200, 40530 Göteborg, Suède, Tél. : 00 46031-773 5254 